\title{
A Wireless Metamaterial-Inspired Passive Rotation Sensor With Submilliradian Resolution
}

\author{
Ali Maleki Gargari, Student Member, IEEE, Burak Ozbey, Member, IEEE, \\ Hilmi Volkan Demir, Senior Member, IEEE, Ayhan Altintas, Senior Member, IEEE, Utku Albostan, \\ Ozgur Kurc, and Vakur B. Ertürk, Senior Member, IEEE
}

\begin{abstract}
A novel passive wireless rotation sensing system with high levels of sensitivity and resolution is proposed and demonstrated for measuring elastic-region bending in materials such as steel. This system is composed of a transceiver antenna and a double-plate sensor in the form of an inter-digital configuration, which does not incorporate any active component. The sensor exhibits a large rotation resolution of $20 \mu$-rad, an excellent sensitivity of $28 \mathrm{MHz} /{ }^{\circ}$ in average, and a large linear dynamic range of approximately $40^{\circ}$. In operation, as a result of the relative rotation between the plates of the sensor, the operating resonance frequency of the system is shifted. This is read out and tracked in the $S_{11}$ response of the transceiver antenna from which the rotation angle is determined. The prototype is designed for microwave regime and it is suitable for measuring very small angles $\left(10^{-4} \sim 10^{-5} \mathrm{rad}\right)$. Critical figuresof-merit of the sensor including sensitivity, dynamic range, and resolution are assessed via systematic measurements, and the validity of resolution experiment is verified by employing digital image correlation method for 2-D measurements.
\end{abstract}

Index Terms - Wireless passive sensing system, rotation sensor, metamaterial-inspired sensor.

\section{INTRODUCTION}

I $\mathrm{N}$ RECENT years, steel construction structures have increasingly become more advanced and popular all around the world thanks to the advantages they bring in the speed and ease of construction. Beams are the major load carrying members in these structures, subjected to bending deformations (in the form of displacement along the load axis, in the form of rotation normal to the loading plane). These beams are

Manuscript received March 3, 2018; accepted March 16, 2018. Date of publication April 3, 2018; date of current version May 9, 2018. The associate editor coordinating the review of this paper and approving it for publication was Dr. Francis P. Hindle. (Corresponding author: Ali Maleki Gargari.)

A. Maleki Gargari and V. B. Ertürk are with the Department of Electrical and Electronics Engineering, Bilkent University, TR-06800 Ankara, Turkey (e-mail: alimaleki@alumni.bilkent.edu.tr; vakur@ee.bilkent.edu.tr).

B. Ozbey is with the ElectroScience Laboratory, Ohio State University, Columbus, OH 43212 USA (e-mail: ozbey.2@osu.edu).

H. V. Demir is with the Department of Electrical and Electronics Engineering and the Department of Physics, Institute of Materials Science and Nanotechnology (UNAM), Bilkent University, TR-06800 Ankara, Turkey, and also with the School of Electrical and Electronic Engineering and the School of Physical and Mathematical Sciences, Nanyang Technological University, Singapore 639798 (e-mail: volkan@stanfordalumni.org).

A. Altintas is with the Communications and Spectrum Management Research Center (ISYAM), Department of Electrical and Electronics Engineering, Bilkent University, TR-06800 Ankara, Turkey (e-mail: altintas@ ee.bilkent.edu.tr).

U. Albostan and O. Kurc are with the Department of Civil Engineering, Middle East Technical University, TR-06800 Ankara, Turkey (e-mail: albostan@metu.edu.tr; kurc@metu.edu.tr).

Digital Object Identifier 10.1109/JSEN.2018.2822671 designed in such a way that deformations remain in the elastic region (i.e., fully reversible) under daily loads. The allowed deformations in such beams are small and usually correspond to rotation of the beam's neutral axis (no deformation axis) in the range of $10^{-4} \sim 10^{-5}$ radians. As a result, in steel constructions, measuring the neutral axis rotations along a beam length is important for obtaining valuable information about the loading, internal force, and deformations building up on the beam. Therefore, critical information regarding the structural health monitoring (SHM) and inspection of the steel constructions can be acquired via rotation sensing. The main challenge here is, however, detecting the rotation due to bending deformations requiring a measurement system with extremely high precision in terms of sensitivity and resolution. To the best of our knowledge, there is no developed technology that employs wireless passive microwave devices for the telemetric measurement of small bending-based deformations with such high sensitivity.

Most of the available rotation sensors are wired and/or composed of active elements such as microwave gyroscopes [1], fiber-optic sensors [2], chirped fiber grating rotation sensor [3], coupled lasers rotation sensor (CLARS) [4], optical fiber rotation sensors [5], and surface acoustic wave (SAW) rotation sensors [6], [7]. In [8], a MEMS high-speed angular sensor has been reported, which can be considered as a semi-active design and is capable of wirelessly measuring the angular movement. Also, passive and wired rotation sensors such as metamaterial-based rotation sensors, incorporating split-ring resonator (SRR) loaded coplanar waveguide (CPW) geometry, have been introduced in [9]-[11]. In these configurations, since the resonance notch depth is dependent on the displacement or rotation of the SRR, this spectral feature can be used to sense the amount of displacement or rotation. In [12] and [13], a tapered diamond shaped SRR and a horn-shaped SRR have been shown using the same operating principle over a fixed resonance frequency, but their dynamic range is limited only to around $6^{\circ} \sim 8^{\circ}$. Similarly, a rotation sensor based on an electric-LC (ELC) resonator coupled CPW has been reported in [14] with a dynamic range up to $90^{\circ}$. A metamaterialinspired rotation sensor has been introduced with an improved dynamic range up to $180^{\circ}$ in [15], and another SRR loaded $\mathrm{CPW}$ sensor based on amplitude modulation of a single-tone continuous wave feeding signal has recently been shown to cover the whole $360^{\circ}$ range [16]. Although some of these metamaterial-based sensors have sufficiently high dynamic 
ranges, they are wired; and their resolution level is not sufficient for measuring small rotations that frequently occur in steel construction.

In this paper, a metamaterial-inspired passive wireless rotation sensing system for the measurement of elastic-region bending deformations in materials such as steel is proposed and demonstrated. The system can be employed in beams made of other elastic materials, composites, plastics, and rubbers as well. The proposed sensing system is designed on the basis of near-field coupling between an antenna and sensor pair, which enhances the key figures-of-merit such as sensitivity, resolution and dynamic range. Thus, the fabricated prototypes manifest high levels of resolution and sensitivity in capturing very slight angular deviations. The organization of the paper is as follows: In Section II, architecture of the proposed sensor is explained. In Section III, all the experiments are described and in Section IV, results and discussion on the experiments are presented.

\section{Architecture of the Sensor}

Our sensing system mainly comprises two elements; a rotation sensor acting as the sensing probe and an external interrogating antenna. The antenna is positioned at the center of the sensor and the distance between them is kept short so that electromagnetic interactions between them is strengthened by near-field coupling leading to a high resolution and sensitivity in a relatively high dynamic range. By this coupling, the resonance frequency of the coupled system can directly be observed in the input impedance of the antenna by a spectrometer such as a vector network analyzer (VNA). The top-and cross-sectional views of the proposed rotation sensor architecture are given in Figs. 1(a) and 1(b), respectively, together with the picture of the top and bottom plates of a fabricated prototype in Fig.1(c). The sensor is composed of two dielectric substrates (top and bottom substrates). On the top of the bottom substrate (as well as on the bottom side of the top substrate) there are metal layers in the form of two quarter-ring comb-like configuration (with four fingers; $N=4$ ) that are electrically connected in the middle (and hence, forming a bow tie shape) as depicted in Fig. 1(a). The comb-like or nested split-ring resonator (NSRR) geometry was first proposed in [17] and used in [18] and [19] as a wireless displacement sensor. The two metal layers of the rotation sensor are facing each other, as shown in Fig. 1(b), to form an inter-digital configuration; however, they are separated by an air-gap, $G_{a}$ which can be filled with grease, if desired. The dielectric substrate is FR-4 with a relative dielectric constant of $\epsilon_{r}=4.3$ and, a thickness of $h_{d}=1.5 \mathrm{~mm}$, where the metal thickness, $h_{m}$, is $18 \mu \mathrm{m}$. The radius of the overall structure is $R$, the finger widths for the top and bottom quarter-rings are denoted with $W$ and $G$, respectively. Thus, the distance between the fingers are $G+2 \times G_{d}$ and, $W+2 \times G_{d}$, respectively, with $G_{d}$ being an extra offset introduced to avoid possible short circuiting of the top and bottom metal layers in the case that $G_{a}$ is not sufficient (i.e., to guarantee an inter-digital configuration between the metal layers). Top and bottom metal layers are, however, electrically connected to each other in the middle via a metallic mandrel that has a

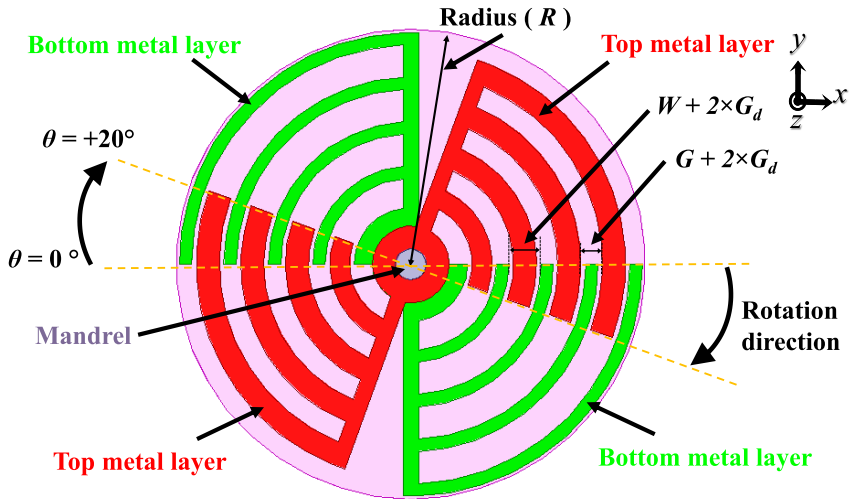

(a)

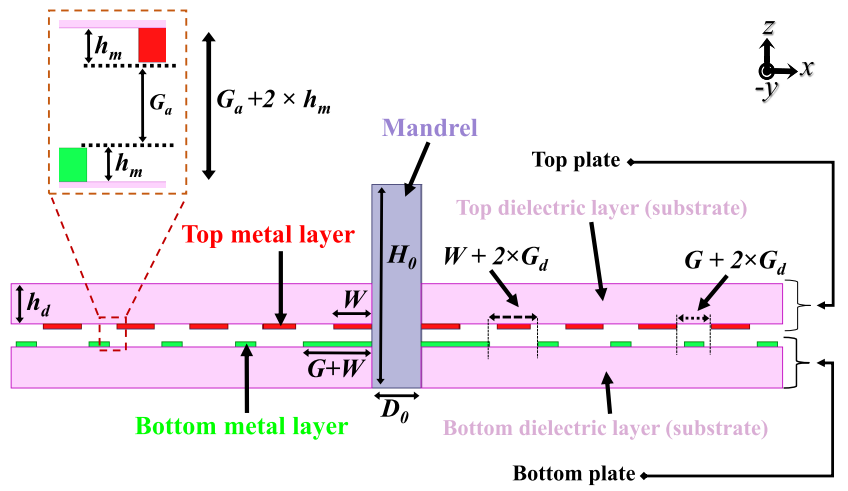

(b)

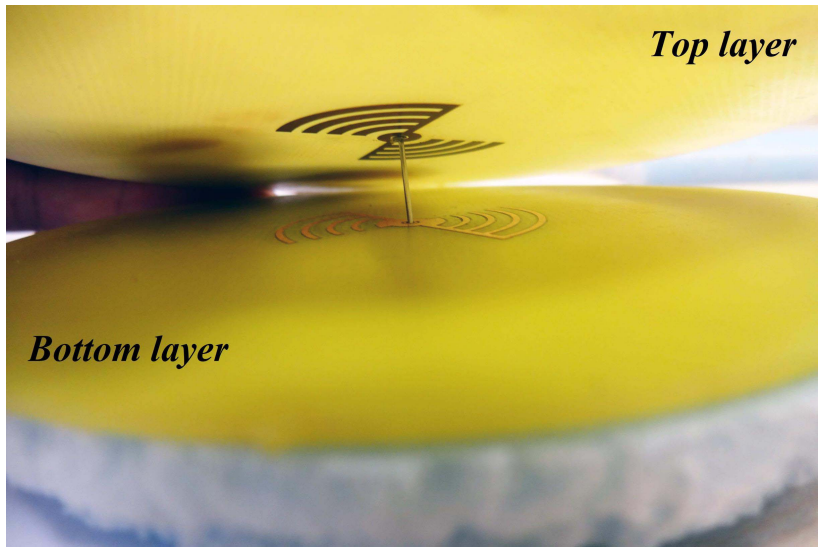

(c)

Fig. 1. Structure of the proposed sensor together with a photograph of its fabricated prototype. (a) Top and bottom metallic layers (at $\theta=20^{\circ}$ ). (b) Side view (at $\theta=20^{\circ}$ ). (c) Top and bottom plates of the fabricated prototype.

diameter of $D_{0}=1 \mathrm{~mm}$ and a height of $H_{0}=4 \mathrm{~mm}$. Final values of $G, W, R, G_{d}$ and $G_{a}$ as well as the number of fingers $(N)$ are locally optimized via Ansoft HFSS to achieve the maximum sensitivity, and are tabulated in Table I.

The operating principle of the sensor is based on the relative rotation of one of the plates with respect to the other (as shown in Fig. 1(a), for example with the $20^{\circ}$ rotation in marked direction) which increases (or decreases) the inter-digitally overlapping area of the two plates. This changes the coupling between the plates, and thus the equivalent $L C$ of the sensor is varied. Consequently, the resonance frequency of the sensor 
TABLE I

Final Values of the Optimized Design Parameters FOR MAXIMUM SENSITIVITY

\begin{tabular}{|c|c|c|c|c|c|}
\hline$N$ & $G$ & $W$ & $R$ & $G_{d}$ & $G_{a}$ \\
\hline 4 & $1.1 \mathrm{~mm}$ & $1.7 \mathrm{~mm}$ & $16.3 \mathrm{~mm}$ & $0.1 \mathrm{~mm}$ & $0.2 \mathrm{~mm}$ \\
\hline
\end{tabular}
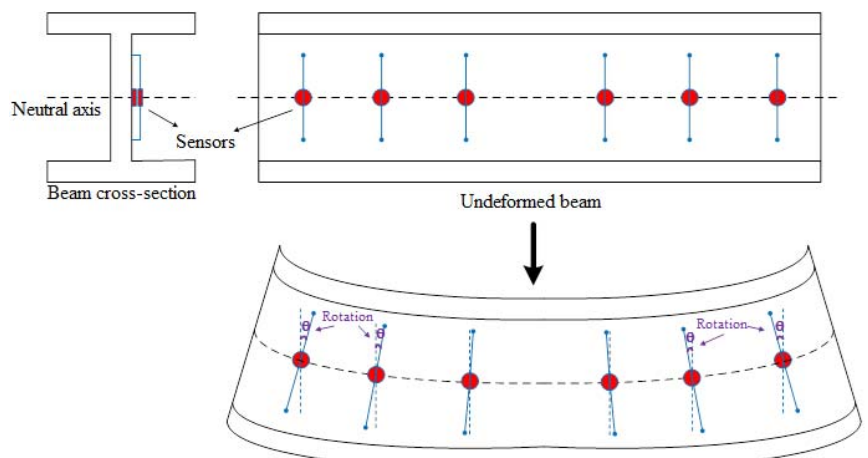

Beam undergone bending deformation

Fig. 2. Diagram illustrating the application of proposed sensors to measure rotation along a structural beam.

is shifted, i.e., decreases if the inter-digitally overlapped area increases and vice versa.

The interrogating antenna used in operation, depicted in the inset of Fig. 3, is a single-slot microstrip antenna based on the design given in [20], and is modified to operate around $2 \mathrm{GHz}$ in this study. It has an approximate bandwidth of $10 \%$ for $\left|S_{11}\right|<10 \mathrm{~dB}$. The slot part is at the front side of the substrate facing the rotation sensor and is fed via a microstrip line at the back of the substrate. The feeding microstrip line is along the $x$-direction resulting an $x$-polarized electric field being transmitted from the slot, which corresponds to $\theta=0^{\circ}$ for the rotation sensor (see Fig. 1(b)).

As discussed in Section I, beams are the major load-carrying members in steel construction structures, which carry these loads along their length by exhibiting: 1) a displacement motion along the direction of the force, and 2) a rotation motion along the direction perpendicular to the direction of the force. According to the elastic beam theory, it can be assumed that the elongation and contraction due to bending are zero along a so-called neutral axis, while increasing linearly away from the neutral axis. Based on this assumption, a possible usage of the suggested sensor can be as follows: The bottom plate of the sensor can be attached at a fixed position on the neutral axis on the body of a bending structure (e.g., a beam), while the top plate is mechanically connected to two points which are positioned above and below the neutral axis and which have equal distances to the neutral axis. Sticks that are rigid enough should be used for this purpose. Then, the contraction at the region above and the elongation at the region below the neutral axis are transformed into a rotation motion at the center of the sensor (angle $\theta$ in Fig. 2). As proposed in [21] and shown in [22] for wireless displacement and strain monitoring of a structural beam, the response from each sensor can be acquired without parasitic coupling in case their central frequencies and bandwidths are set so as not to have a spectral overlap. Hence, by positioning

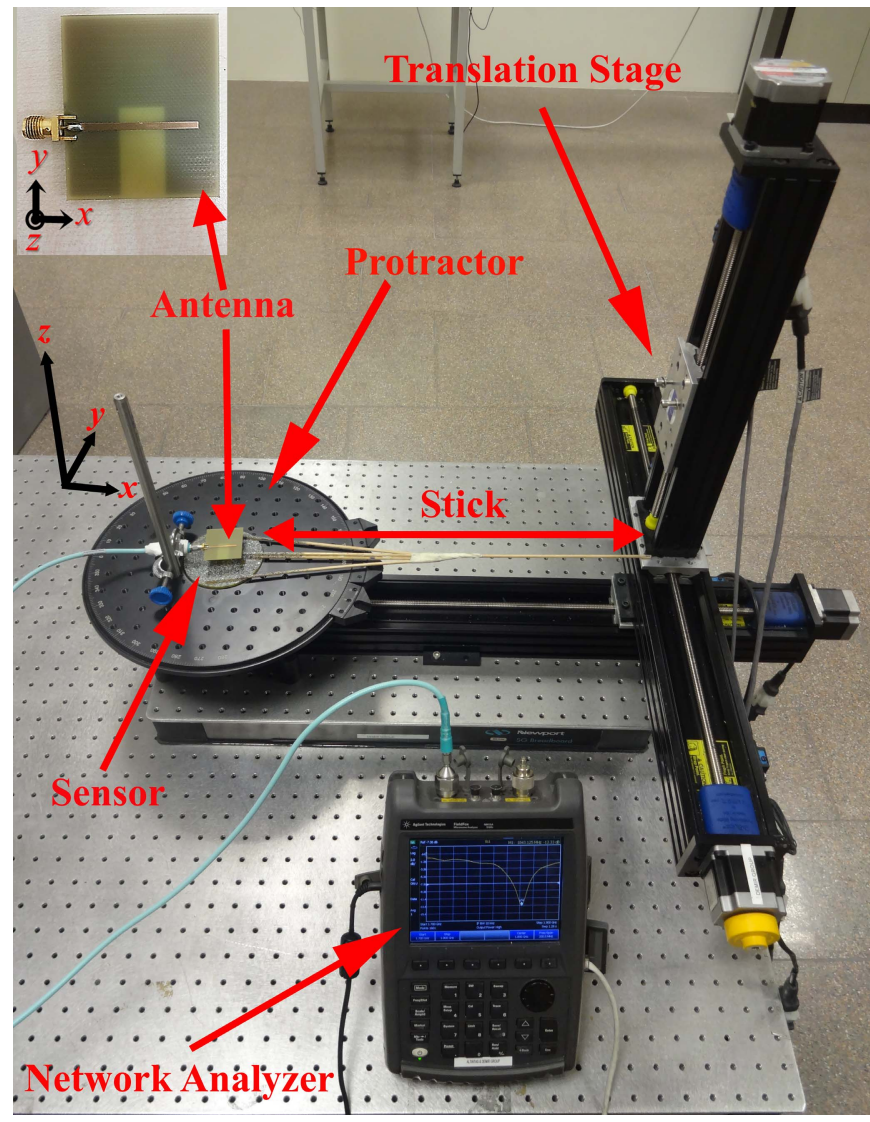

Fig. 3. Experiment setup for the measurement of frequency change as a function of the rotation angle, with the antenna zoomed in the inset.

multiple sensors along the beam, response from each sensor can either be acquired at once or at different time instants. This way, the change of rotation along the entire length of the beam can be obtained. This information can then be converted into transverse displacement using the elastic beam theory.

\section{EXPERIMENTS}

Two sets of experiments were performed with the rotation sensor to assess its figures-of-merit using the experiment setup shown in Fig. 3. A fork-like configuration with a length of $L_{s}=50 \mathrm{~cm}$ (all sticks having a diameter of $1 \mathrm{~cm}$ ) was applied to ensure more stable rotation by increasing the bending stiffness of the sticks during the measurements. The fork part was fixed on the top dielectric layer of the sensor while its handle part was connected to the moving part of the translation stage with flexible adhesive (blu-tack). Therefore, a precisely controlled linear movement of the translation stage was converted to a micron-level controlled-rotation on the sensor. Also, WD-40 brand grease was applied to the air gap, $G_{a}$, between the top and bottom metallic layers to minimize the friction between top and bottom plates of the sensor. In the first set of experiments, linearity, sensitivity, dynamic range, and resolution of the sensor were assessed by rotating the top plate of the sensor (while its bottom plate was fixed on the protractor) and recording the shifts in the resonance frequency of the coupled system via a VNA for different rotation angles. 
For the second set of experiments, a parameter named rotation tracking range was defined in a fashion similar to [18] and its variation with respect to the distance between the antenna and the sensor (called monitoring distance, $D_{m}$ ) was explored. Note that the center part of the protractor, over which the sensor was positioned, is removable. Hence, experiments were first performed in its absence, where the sensor was mounted on a dielectric (Styrofoam) layer. Then, experiments were repeated when the sensor was mounted on a metallic sheet so that its effects on the aforementioned figures-of-merit could be investigated.

\section{RESUltS AND DisCUSSION}

\section{A. Sensor Characterization}

The resonance of the coupled system manifests itself in $\left|S_{11}\right|$ in the form of a dip as depicted in Figs. 4(a) and 4(b) for the sensor positioned on a dielectric (Styrofoam) layer and a metallic sheet, respectively. The shifts in the resonance frequency can be clearly seen as the sensor was rotated from $5^{\circ}$ to $45^{\circ}$ with $5^{\circ}$ steps. As expected, by increasing the rotation angle $(\theta)$, the interdigital area is expanded and the equivalent capacitance of the system is accordingly increased, which yields a decrease in the resonance frequency. Fig. 4(c) presents these resonance frequency shifts of the coupled system versus rotation angle $(\theta)$ for both experiment and simulation results when the sensor was on the dielectric layer and on the metallic sheet. In Fig. 4(c), the experiment data are the average of ten different measurements for each case, and the simulations are carried out in Ansoft HFSS. A reasonable agreement between the measurements and the simulations are observed. Exclusion of grease in the simulations, manufacturing tolerances and some uncertainties in some dimensions (in particular, the airgaps, $G_{a}$ and $G_{d}$ ) are considered as the potential sources of discrepancies especially in small $\theta$ values. According to the results presented in Figure 4(b), spurious dips are observed at rotation angles equal to $25^{\circ}, 35^{\circ}$, and $45^{\circ}$. For $25^{\circ}$ and $35^{\circ}$, there is an observable amplitude difference between two dips, and following the one that has the largest dip would give accurate measurements. On the other hand, if the initial rotation is set to $45^{\circ}$ and the measurements are taken at discrete time intervals, such spurious responses may cause measurement errors. However, it should be kept in mind that $45^{\circ}$ is just at the limit of the linear dynamic range of this sensor as will be discussed next.

Linearity of the sensor is assessed using the coefficient of determination, i.e., $R^{2} . R^{2}$ is a statistical parameter that quantifies the deviation of an actual curve from a fitted linear curve. It varies between 0 and 1, 1 implying perfect linearity. The definition of this parameter is given in Appendix V. The $R^{2}$ value is found to be over 0.99 in every $5^{\circ}$ rotation range as tabulated in Table II and Table III, for the dielectric layer and metallic sheet cases, respectively, indicating not only high linearity but also exhibiting additional advantage of the sensor that it can be operated starting from different initial rotation angles without sacrificing its linear response. Simply, allowing an initial $\theta$ on the sensor to create a frequency offset, the sensor can be employed in higher rotation angle operation regions depending on the linearity and the resolution of that

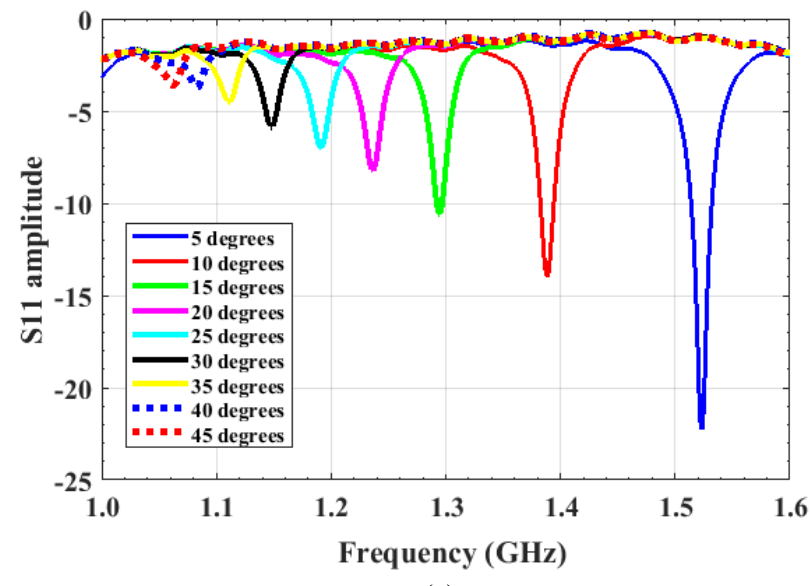

(a)

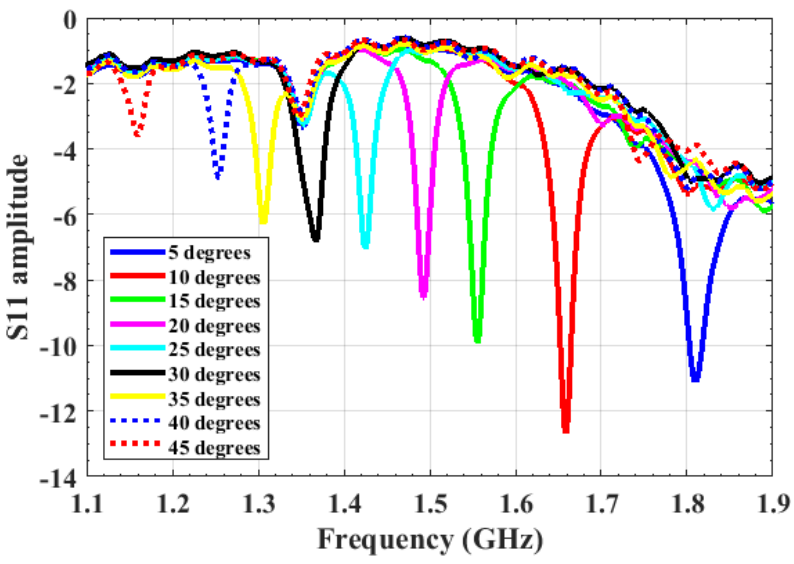

(b)

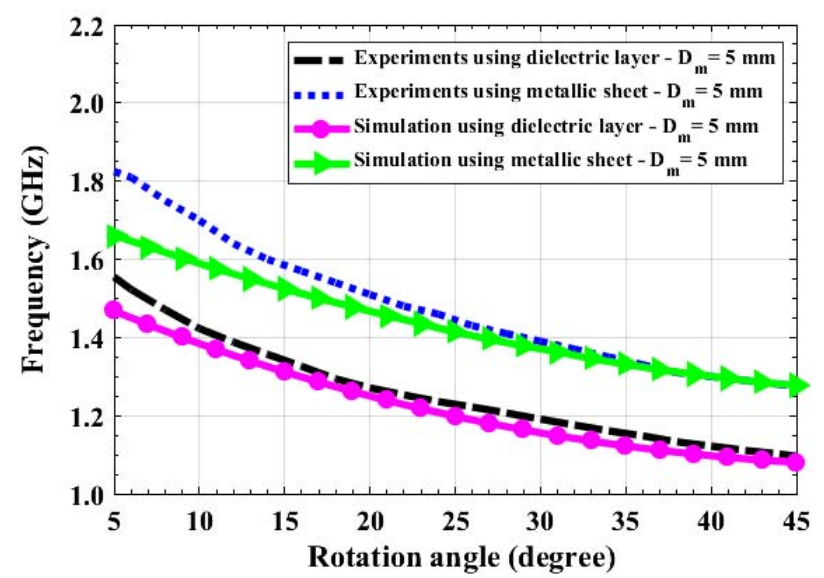

(c)

Fig. 4. (a) Experimental $\left|S_{11}\right|$ data versus frequency showing the shifting of the resonance frequency (in the form of dips) for different rotation angles $\theta$ for the dielectric (i.e., Styrofoam) case. (b) Experimental $\left|S_{11}\right|$ curves versus frequency that shows the shifting of the resonance frequency (in the form of dips) for different rotation angles $\theta$ for the metallic sheet case. (c) The shift in the resonance frequency (in the form of dips) of the coupled system versus the rotation angle $\theta$.

region of choice. Sensitivity, which describes the spectral change read out telemetrically from the sensor in response to the rotation, can be mathematically defined as the slope of the frequency-rotating angle curve, presented in Fig. 4(c) with 
TABLE II

EXPeriment Results of the SEnsitivity Test And $R^{2}$ Parameter FOR SEVERAL SELECTED ROTATION RANGES FOR THE DieleCtric LAYER (STYROFOAM) CASE

\begin{tabular}{|c|c|c|}
\hline \multicolumn{3}{|c|}{ Over Styrofoam } \\
\hline Rotation range $\left(^{\circ}\right)$ & Sensitivity $(\mathbf{M H z} / \mathbf{d e g})$ & $R^{2}$ \\
\hline 5 to 10 & 26.4 & 0.99 \\
\hline 10 to 15 & 19.28 & 0.99 \\
\hline 15 to 20 & 15.61 & 0.99 \\
\hline 20 to 25 & 9.35 & 0.99 \\
\hline 25 to 30 & 7.94 & 0.99 \\
\hline 30 to 35 & 6.63 & 0.99 \\
\hline 35 to 40 & 6.07 & 0.99 \\
\hline 40 to 45 & 5.68 & 0.99 \\
\hline
\end{tabular}

TABLE III

EXPERIment Results of the SEnSitivity Test and $R^{2}$ PARAmeter FOR SEVERAL SELECTED ROTATION RANGES FOR The Metallic Sheet CASE

\begin{tabular}{|c|c|c|}
\hline \multicolumn{3}{|c|}{ Over metallic sheet } \\
\hline Rotation range $\left(^{\circ}\right)$ & Sensitivity (MHz/deg) & $R^{2}$ \\
\hline 5 to 10 & 28.8 & 0.99 \\
\hline 10 to 15 & 23.2 & 0.99 \\
\hline 15 to 20 & 17.07 & 0.99 \\
\hline 20 to 25 & 12.88 & 0.99 \\
\hline 25 to 30 & 10.84 & 0.99 \\
\hline 30 to 35 & 8.59 & 0.99 \\
\hline 35 to 40 & 7.49 & 0.99 \\
\hline 40 to 45 & 6.64 & 0.99 \\
\hline
\end{tabular}

a unit of MHz/degree. The sensitivity results of the sensor are also tabulated in Table II and Table III for both dielectric layer and metallic sheet cases, respectively, for various $5^{\circ}$ rotation ranges. As can be seen from the tables (as well as from Fig. 4(c)), sensitivity of the sensor is very high at low rotation angles for both cases $(26.4 \mathrm{MHz} / \mathrm{deg}$ for the dielectric case and $28.8 \mathrm{MHz} / \mathrm{deg}$ for the metallic sheet case for the $5^{\circ} \leq$ $\theta \leq 10^{\circ}$ interval) but decreases at higher rotation angles (though $R^{2}$ is still over 0.99 ). On the other hand, linearity also degrades as the rotation range is increased. Therefore, instead of investigating piecewise linearity, one can check the maximum linear range of the proposed sensing system. Defining the linear dynamic range as the maximum rotation range that can be read out under the constraint that $R^{2}>0.95$, the linear dynamic range of the proposed rotation sensor is approximately $40^{\circ}$. Consequently, an optimum rotation range can be selected subject to limitations by the structure whose bending based rotation to be sensed as well as the desired operation frequency.

Another important figure-of-merit for a sensor is its resolution, and it was experimentally demonstrated that the proposed rotation sensor is sensitive to $\mu$-radian rotation levels. In this work, the resolution is defined as the minimum level of rotation that can be measured given the system noise. Fig. 5 and Fig. 6 illustrate the resolution test over dielectric and metallic sheet, respectively, for a range of $12000 \mu$-radians. During the experiment, the first $8000 \mu$-radian rotation was applied with $40 \mu$-radian step size (i.e., the first 200 steps) and the next $4000 \mu$-radian rotation was applied with $20 \mu$-radian step size (i.e., the next 200 steps). After each step, the translation stage was paused for 8 seconds for data collection. The data

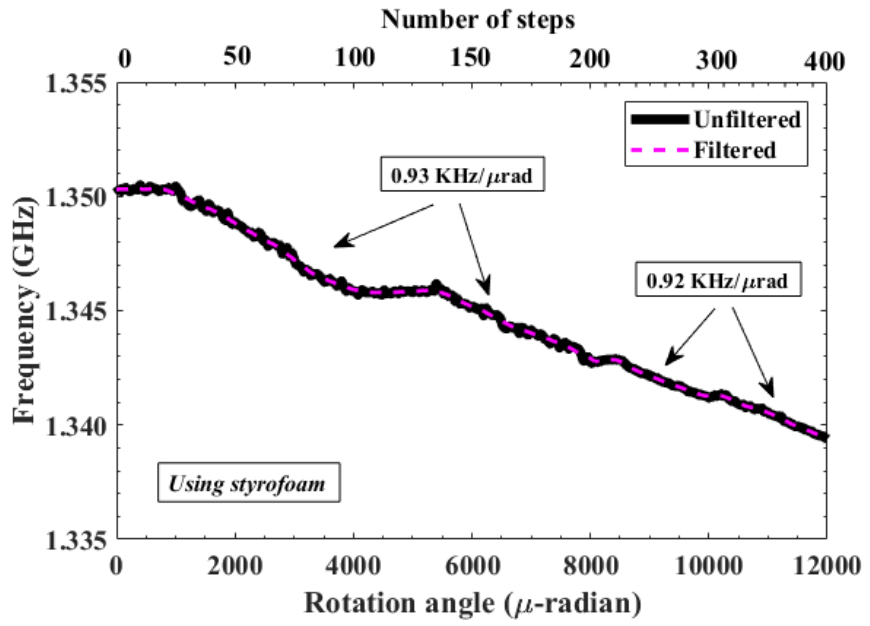

Fig. 5. Resolution test (frequency versus $\mu$-radians rotation) when the sensor is over dielectric (Styrofoam). First 200 steps were applied with $40 \mu$-radian step size (up to $8000 \mu$-radians) and the next 200 steps were applied with $20 \mu$-radian step size (between $8000 \mu$-radians and $12000 \mu$-radians). Experiment was paused for 5 minutes after every 100 steps.

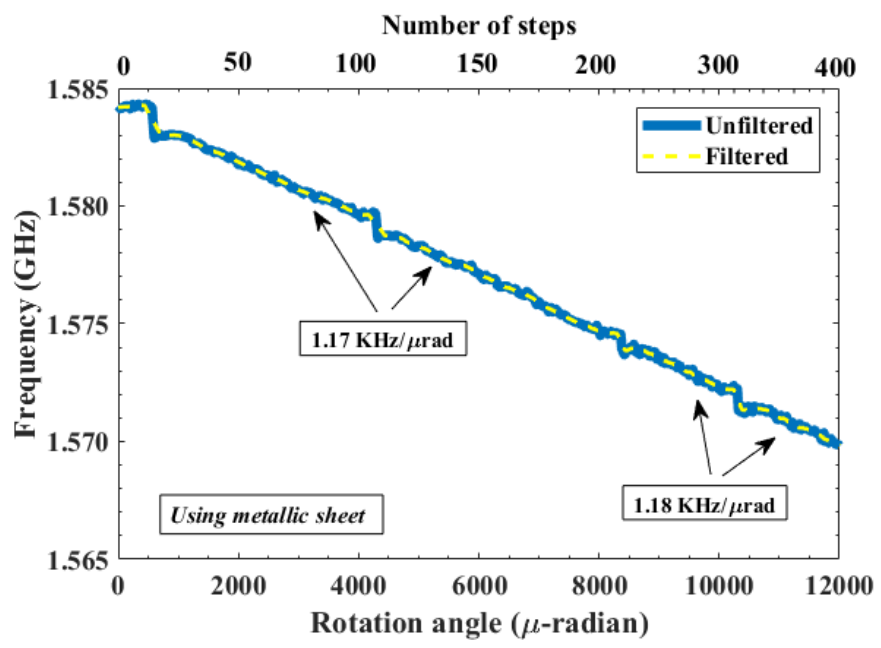

Fig. 6. Resolution test (frequency versus $\mu$-radians rotation) when the sensor is over metallic sheet. First 200 steps were applied with $40 \mu$-radian step size (up to $8000 \mu$-radians) and the next 200 steps were applied with $20 \mu$-radian step size (between $8000 \mu$-radians and $12000 \mu$-radians). Experiment was paused for 5 minutes after every 100 steps.

used to generate both figures (i.e., Fig. 5 and Fig. 6) were taken with the IF bandwidth set to $1 \mathrm{kHz}$ together with the averaging option of the VNA without any smoothing so that the system noise was kept. After the data acquisition the raw data was filtered (with a low-pass filter) to remove the highfrequency fluctuations. Both filtered and unfiltered data are depicted in Fig. 5 and Fig. 6. In these figures, when the portion of the curves between the 1000-2000 $\mu$-radian is considered, it is possible to see a continuous change at a large slope, which shows the response of the sensor, implying high sensitivity. In this portion of the curve where a total of 1 milliradian of rotation takes place, there are 25 data points, and they show very little deviation, especially for the filtered signal. This finding definitely indicates a sub-milliradian resolution, which is probably in the order of 10 s to 100 s of microradian. 


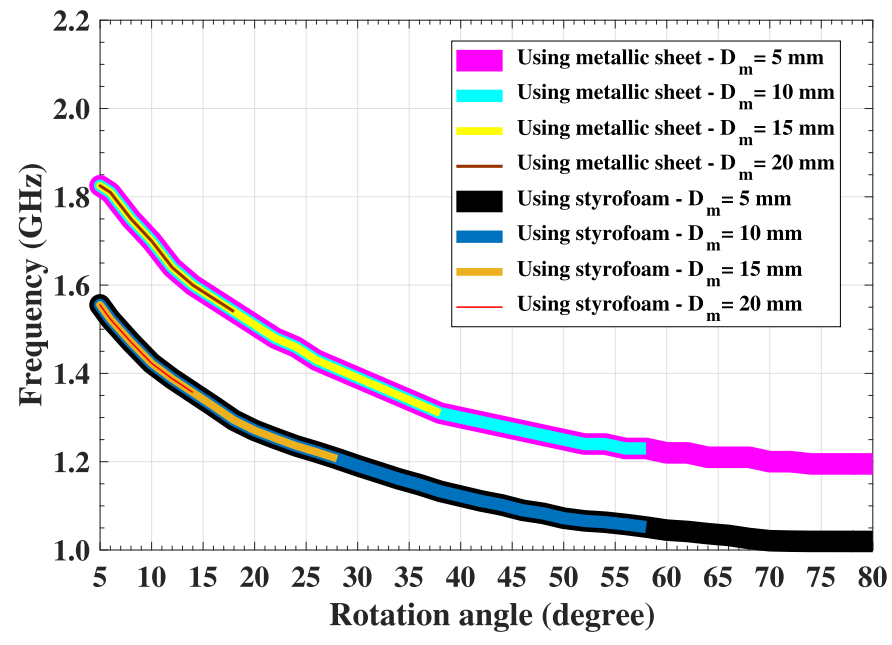

Fig. 7. Rotation tracking range variation for different monitoring distances $D_{m}$ for a tracking threshold of $1 \mathrm{~dB}$.

Even though it is not easy to figure the exact resolution value, these findings definitely indicate a submilliradian resolution, which is probably in the order of 10 s to 100 s of $\mu$-radian.

After every 100 steps, the experiment was paused for about 5 minutes. It can be seen in both Fig. 5 and Fig. 6 that there is almost a flat portion with no frequency change immediately after the pause. Then, especially in metallic sheet case, there is a sudden frequency jump and afterwards frequency varies almost linearly with the variation of the rotation. The flat portion in both figures can be due to two reasons: Either the motion of the translation stage is not fully transferred to the sensor plate through the wooden sticks or the mandrel detaches from the movable top plate, and both plates become electronically disconnected. As more rotation is applied to the system through the translation stage, a sudden change in the slope is observed which indicates the aforementioned problems are resolved and the sensor operates accurately.

At regions where the sensor operates accurately, a linear relationship between frequency change and rotation angle can be observed for both dielectric and metallic sheet cases. Considering the first 200 steps (up to $8000 \mu$-radians) and the next 200 steps (from $8000 \mu$-radians to $12000 \mu$-radians), the slopes of the two linear regions in each group of 200 steps are relatively the same. The slope of every linear region (given in $\mathrm{KHz} / \mathrm{rad}$ ) is displayed accordingly in both Fig. 5 and Fig. 6. It should be noted that the resolution test for the dielectric case was performed somewhere in the interval of $13^{\circ}<\theta<15^{\circ}$ whereas it was performed somewhere in the interval of $15^{\circ}<\theta<17^{\circ}$ for the metallic sheet case. Therefore, considering these intervals, the slopes of the linear regions are also consistent with previously presented sensitivity results. Besides, it has been observed that having a metallic sheet underneath the bottom plate of the sensor increased the operating frequency of the sensor and the sensitivity.

The rotation tracking range, defined as the maximum rotation range that can be captured by the antenna, was investigated in the second set of experiments in a fashion similar to that described in [18] and [19]. Fig. 7 illustrates the rotation tracking range of the rotation sensor for various monitoring
TABLE IV

Comparison of the Performance of the Proposed SENSOR With OTHER PASSIVE Rotation SENSORS PREVIOUSLY REPORTED IN LITERATURE

\begin{tabular}{|l|l|l|l|l|}
\hline Sensor type & $\begin{array}{l}\text { Wireless } \\
\text { or wired }\end{array}$ & Resolution & Sensitivity & $\begin{array}{l}\text { Dyn. } \\
\text { range }\end{array}$ \\
\hline $\begin{array}{l}\text { SRR loaded } \\
\text { CPW [13] }\end{array}$ & Wired & No info & $\begin{array}{l}\text { Notch depth shift } \\
\text { based: 2dB/deg }\end{array}$ & $7^{\circ}$ \\
\hline $\begin{array}{l}\text { ELC loaded } \\
\text { CPW [14] }\end{array}$ & Wired & No info & $\begin{array}{l}\text { Notch depth shift } \\
\text { based: 2dB/deg }\end{array}$ & $90^{\circ}$ \\
\hline $\begin{array}{l}\text { Tapered } \\
\text { U-shaped [15] }\end{array}$ & Wired & No info & $\begin{array}{l}\text { Freq. shift based: } \\
1.8 \mathrm{MHz} / \mathrm{deg}\end{array}$ & $180^{\circ}$ \\
\hline $\begin{array}{l}\text { Chirped fiber } \\
\text { grating [3] }\end{array}$ & Wired & $1^{\circ}$ & $\begin{array}{l}\text { Wavelength shift } \\
\text { based: } 11.7 \mathrm{~nm} / \mathrm{deg}\end{array}$ & $360^{\circ}$ \\
\hline $\begin{array}{l}\text { SRR loaded } \\
\text { CPW [16] }\end{array}$ & Wired & $<1.5^{\circ}$ & No info & $360^{\circ}$ \\
\hline This work & Wireless & $\begin{array}{l}<100 \mu- \\
\text { radian }\end{array}$ & $\begin{array}{l}\text { Frequency shift } \\
\text { based: 28 MHz/deg }\end{array}$ & $40^{\circ}$ \\
\hline
\end{tabular}

distances $\left(D_{m}\right)$ when the sensor is on the dielectric layer (Styrofoam) and on a metallic sheet for a tracking threshold of $1 \mathrm{~dB}$ [18]. It should be pointed out that the one-to-one relationship between the rotation angle and the corresponding resonance frequency is independent of the antenna's position with respect to the sensor; that is, the measured shift in the resonance frequency of the coupled system (appearing in the form of a dip in $\left|S_{11}\right|$ spectrum) corresponds to the same rotation angle. However, the rotation tracking range decreases as the monitoring distance is increased. When $D_{m}$ is $5 \mathrm{~mm}$, the entire $75^{\circ}$ rotation range can be tracked by the sensor for the aforementioned tracking threshold of $1 \mathrm{~dB}$. However, for the same tracking threshold value, it reduces nearly to $10^{\circ}$ when $D_{m}$ is $20 \mathrm{~mm}$. One can reduce the tracking threshold value to improve the monitoring distance, though it is limited by the system noise.

The performance of the proposed sensor is compared to other passive rotation sensors shown in literature in Table IV. As shown, the proposed sensor is the only real wireless passive sensor yet available in literature. Also, it demonstrates a better resolution and a good sensitivity, making it ideal for the SHM applications.

\section{B. Digital Image Correlation}

In order to understand the behavior of the sensor after each pause (flat regions in both Fig. 5 and Fig. 6), the experiment for the dielectric case was repeated with a step size of $80 \mu$-radians and rotations were also measured with digital image correlation (DIC) method to observe the actual rotation of the sensor plates. DIC method utilizes successively taken digital images to compute displacement and deformation distribution through the whole surface without any contact to the specimen [24]. For this purpose, the top surface of the sensor plate was painted in such a way that it had a random grayscale color distribution. Usually a speckled pattern is utilized for this purpose (Fig. 8(a)). Then, the images were taken with a high resolution camera and the selected points, named as raster points, were located at every image by utilizing the stochastic information of images. This stochastic information was obtained by considering the intensity values of the raster points and their neighborhood pixels. As the location of a 


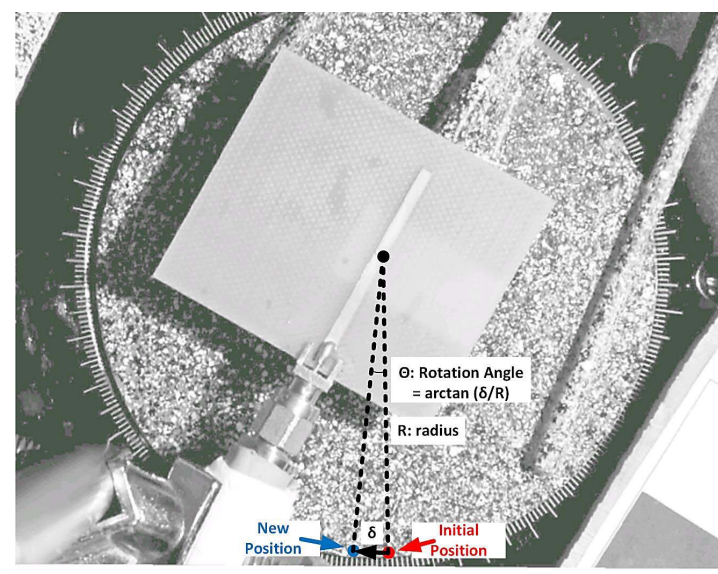

(a)

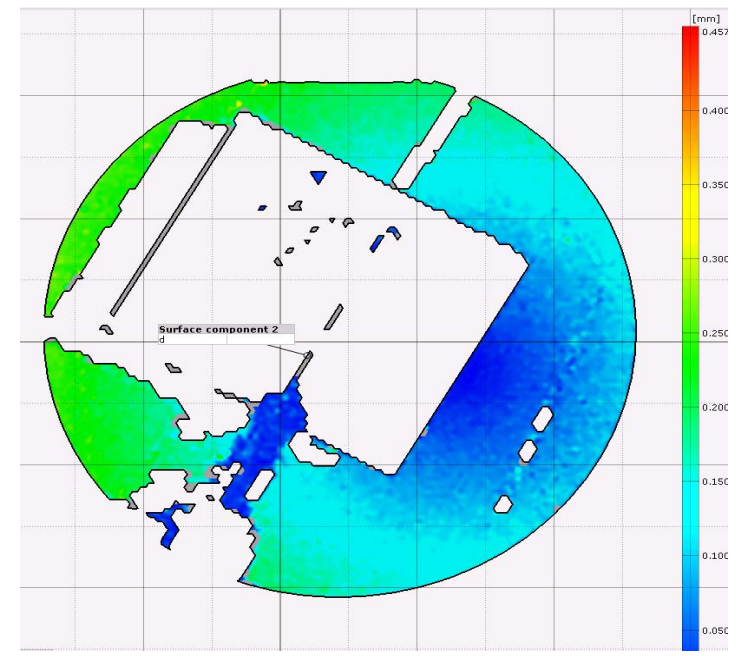

(b)

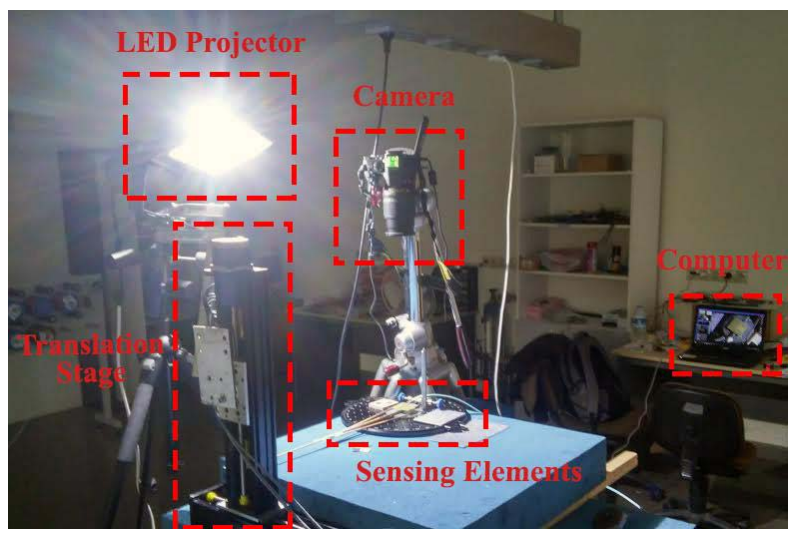

(c)

Fig. 8. (a) Speckled pattern of the surface of the sensors top plate. The location of the raster point is also shown in this figure. (b) An example surface displacement distribution of the top plate. (c) Experiment setup for the DIC method.

raster point at a digital image is determined, the displacement of that point can be computed by comparing its location with the initial or the previous images. Using several raster points at a surface would allow computation of the displacement and deformation of the whole surface (Fig. 8(b)). Fig. 8(c) presents the DIC measurement set-up. A digital camera, Nikon D5200, was placed at the top of the sensor plate in such a way that the

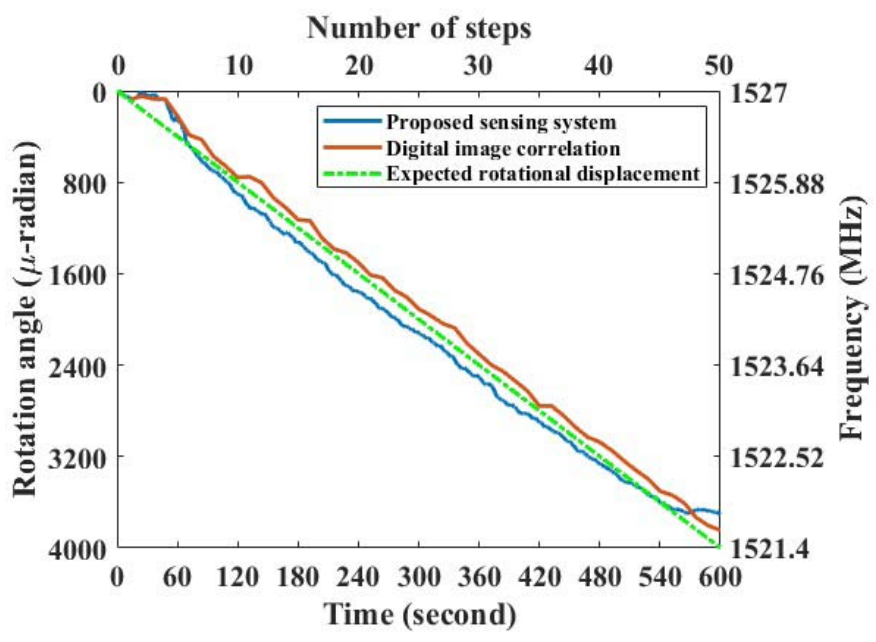

Fig. 9. Comparing the experiment results of the proposed sensing method with the DIC method for a $80 \mu$-radian resolution level, shown along with the expected rotational displacement generated by the moving translation stage.

horizontal plane of the camera sensor became perpendicular to the surface normal of the sensor plate. The surface of the plate was illuminated with an LED projector light source which caused less noise on the camera sensor. The camera was connected to a computer which controlled the image acquisition process. The digital images were acquired as video due to having a small region of interest $(4 \mathrm{~cm}$ in radius) and rather fast movement. The camera has the ability to shoot 59.94 frames per second with 2 MP resolution in video mode which means that $80 \mu \mathrm{m}$ is represented by 1 pixel on the camera sensor. A total of 71,934 frames in 20 minutes were taken during the experiment. Then, the images were extracted from video such that 30 frames were obtained for each one second interval. These 30 frames were averaged geometrically in order to minimize the noise on the images. In the end, 1,200 averaged images were obtained. The averaged images were correlated by using GOM Correlate software (GOM Testing, [25]). One raster point having 9 pixels subset size was chosen on outer edge of the wireless sensor (Fig. 8(a)) and resultant displacement of this point was converted to the rotation angle for each step.

Fig. 9 presents the rotation angle measurement with the proposed sensing system and DIC method. In this experiment, the sensor was placed on the dielectric layer and translation stage was moved in such a way that it generated $80 \mu$-radian rotation at every step. The sensor frequency shifts were converted into rotation angle by using the translation stage data which corresponded to $1.43 \mathrm{KHz} / \mu \mathrm{rad}$. For this purpose the sensor data obtained between $10^{\text {th }}$ and $40^{\text {th }}$ steps were utilized. Both DIC measurement and sensor reading show a flat portion at the beginning of the experiment up to the $4^{\text {th }}$ step. This indicates that the motion of the translation stage was not fully transferred to the sensor plate through the wooden sticks, most probably due to the internal energy required to deform the flexible adhesive or overcome the static friction between the sensor plates. Thus, the flat portion of the sensor readings is mainly because of the insufficiency of the experiment set-up. After the $7^{\text {th }}$ step, a linear behavior is 
observed at two different measurements, which proves that the sensing system is capable of measuring $\mu$-radian level rotations.

\section{CONClusion}

A metamaterial-inspired passive wireless rotation sensing system is proposed and demonstrated for measuring very small elastic-region bending in steel and other elastic materials. The system is composed of a transceiver antenna and a sensor, and is designed on the basis of their near-field coupling to enhance important figures-of-merit including sensitivity, resolution, linearity and dynamic range. The fabricated prototypes exhibit a high linearity $\left(R^{2}>0.99\right.$ for over $5^{\circ}$ rotation range), an excellent sensitivity (approximately $28 \mathrm{MHz} /$ degree when the sensor is on a metallic sheet) together with a large linear dynamic range of $40^{\circ}$ and a high rotation resolution of $20 \mu$-radians. Besides, the resolution performance of the sensor is validated by using DIC method at the limit of the camera system (which was $80 \mu$-radians in our setup). These findings indicate that the proposed rotation sensor holds a great promise for industrial applications.

\section{APPENDIX COEFFicient of Determination $\left(R^{2}\right)$}

If a linear curve is fitted to a set of observed data points, these data points are denoted as $y_{i}$, the fitted value as $\hat{y}_{i}$, and the mean of the observed data as $\bar{y}$, then SSR, SST and $R^{2}$ can be defined as below:

$$
\begin{aligned}
\mathrm{SSR} & =\sum_{i}\left(\hat{y}_{i}-\bar{y}\right)^{2}, \\
\mathrm{SST} & =\sum_{i}\left(y_{i}-\bar{y}\right)^{2}, \\
\mathrm{R}^{2} & =\frac{\mathrm{SSR}}{\mathrm{SST}} .
\end{aligned}
$$

\section{REFERENCES}

[1] G. G. Karapetyan. (2000). "Microwave gyroscope-novel rotation sensor." [Online]. Available: https://arxiv.org/abs/physics/0011014

[2] V. Budinski and D. Donlagic, "Fiber-optic sensors for measurements of torsion, twist and rotation: A review," Sensors, vol. 17, no. 3, p. 443, 2017.

[3] H.-J. Sheng, W.-F. Liu, and M.-Y. Fu, " $360^{\circ}$ rotation sensor using a chirped fiber grating," IEEE Photon. Technol. Lett., vol. 26, no. 2, pp. 162-164, Jan. 15, 2014.

[4] J. Scheuer and B. Z. Steinberg, "Coupled lasers rotation sensor (CLARS)," J. Lightw. Technol., vol. 26, no. 23, pp. 3803-3810, Dec. 1, 2008.

[5] W. K. Burns, P. F. Liao, and P. Kelley, Optical Fiber Rotation Sensing. San Diego, CA, USA: Academic, 2012.

[6] W. Yi-Qun, L. Wei, and Z. Jia-Lin, "A difference SAW rotation sensor," in Proc. IEEE Int. Conf. Inf. Acquisition, Aug. 2006, pp. 866-870.

[7] H. Tiersten, D. Stevens, and P. Das, "Acoustic surface wave accelerometer and rotation rate sensor," in Proc. Ultrason. Symp., Nov. 1980, pp. 692-695.

[8] W. Sun and W. J. Li, "MEMS high-speed angular-position sensing system with RF wireless transmission," Proc. SPIE, vol. 4334, pp. 244-252, Aug. 2001.

[9] S. Harnsoongnoen and N. Angkawisittpan, "Angular displacement sensor based on coplanar waveguide (CPWs) loaded with S-shaped golden spiral-tapered split ring resonators (SGS-SRRs)," Proc. Comput. Sci., vol. 86, pp. 75-78, Jan. 2016.

[10] J. Naqui and F. Martín, "Angular displacement and velocity sensors based on electric-LC (ELC) loaded microstrip lines," IEEE Sensors J., vol. 14, no. 4, pp. 939-940, Apr. 2014.
[11] J. Naqui, M. Durán-Sindreu, and F. Martín, "Alignment and position sensors based on split ring resonators," Sensors, vol. 12, no. 9, pp. 11790-11797, 2012.

[12] A. K. Horestani, C. Fumeaux, S. F. Al-Sarawi, and D. Abbott, "Displacement sensor based on diamond-shaped tapered split ring resonator," IEEE Sensors J., vol. 13, no. 4, pp. 1153-1160, Apr. 2013.

[13] A. K. Horestani, D. Abbott, and C. Fumeaux, "Rotation sensor based on horn-shaped split ring resonator," IEEE Sensors J., vol. 13, no. 8 , pp. 3014-3015, Aug. 2013.

[14] J. Naqui and F. Martín, "Transmission lines loaded with bisymmetric resonators and their application to angular displacement and velocity sensors," IEEE Trans. Microw. Theory Techn., vol. 61, no. 12, pp. 4700-4713, Dec. 2013.

[15] A. Ebrahimi, W. Withayachumnankul, S. F. Al-Sarawi, and D. Abbott, "Metamaterial-inspired rotation sensor with wide dynamic range," IEEE Sensors J., vol. 14, no. 8, pp. 2609-2614, Aug. 2014.

[16] J. Mata-Contreras, C. Herrojo, and F. Martín, "Application of split ring resonator (SRR) loaded transmission lines to the design of angular displacement and velocity sensors for space applications," IEEE Trans. Microw. Theory Techn., vol. 65, no. 11, pp. 4450-4460, Nov. 2017.

[17] R. Melik et al., "Nested metamaterials for wireless strain sensing," IEEE J. Sel. Topics Quantum Electron., vol. 16, no. 2, pp. 450-458, Mar. 2010.

[18] B. Ozbey et al., "Wireless displacement sensing enabled by metamaterial probes for remote structural health monitoring," Sensors, vol. 14, no. 1, pp. 1691-1704, 2014.

[19] B. Ozbey, H. V. Demir, O. Kurc, V. B. Erturk, and A. Altintas, "Wireless measurement of elastic and plastic deformation by a metamaterial-based sensor," Sensors, vol. 14, no. 10, pp. 19609-19621, 2014.

[20] S. I. Latif, L. Shafai, and S. K. Sharma, "Bandwidth enhancement and size reduction of microstrip slot antennas," IEEE Trans. Antennas Propag., vol. 53, no. 3, pp. 994-1003, Mar. 2005.

[21] B. Ozbey, V. B. Ertürk, O. Kurc, A. Altintas, and H. V. Demir, "Multi-point single-antenna sensing enabled by wireless nested splitring resonator sensors," IEEE Sensors J., vol. 16, no. 21, pp. 7744-7752, Nov. 2016.

[22] B. Ozbey, V. B. Erturk, H. V. Demir, A. Altintas, and O. Kurc, "A wireless passive sensing system for displacement/strain measurement in reinforced concrete members," Sensors, vol. 16, no. 4, p. 496, Apr. 2016.

[23] D. Vicari, A Note on the Coefficient of Determination (Dipartimento di statistica, probabilità e statistiche applicate), A. Ricerche, Ed. Rome, Italy: Univ. degli studi di Roma La Sapienza, 1996.

[24] M. A. Sutton, J. H. Yan, V. Tiwari, H. W. Schreier, and J. J. Orteu, "The effect of out-of-plane motion on 2D and 3D digital image correlation measurements," Opt. Lasers Eng., vol. 46, no. 10, pp. 746-757, 2008.

[25] (Nov. 16, 2016). GOM Testing Technical Documentation as of V8 SR1, Digital Image Correlation and Strain Computation Basics. Accessed: Jun. 13, 2016. [Online]. Available: http://support.gom.com/ display/DOCS/Digital+Image+Correlation+and+Strain+Computation+ Basics

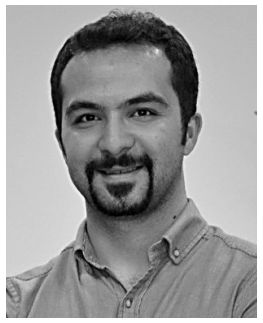

Ali Maleki Gargari received the B.Sc. degree in electrical and computer engineering from the University of Tabriz, Tabriz, Iran, in 2014, and the M.Sc. degree in electrical and electronic engineering from Bilkent University, Ankara, Turkey, in 2017. $\mathrm{He}$ is currently pursuing the Ph.D. degree with The University of British Columbia, Okanagan Campus, Kelowna, BC, Canada. His recent research activities have focused on the design and testing of wireless RF sensors, high impedance electromagnetic surfaces, novel PBG materials, and their applications in microwave-microfluidic systems. He was a recipient of the 2015 IEEE AP-S Eugene F. Knott Memorial Pre-Doctoral Research Grant for "A Wireless Metamaterial-Inspired Passive Rotating Sensing System for Remote Structural Health Monitoring." 


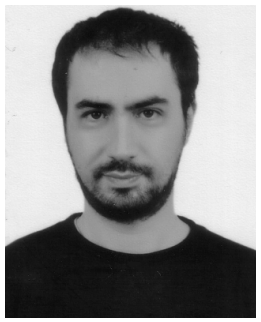

Burak Ozbey (M'16) received the B.S., M.S., and Ph.D. degrees in electrical and electronics engineering from Bilkent University, Ankara, Turkey, in 2008, 2011, and 2016, respectively. He is currently a Post-Doctoral Researcher at the ElectroScience Laboratory, Ohio State University. His research interests include microwave and $\mathrm{THz}$ systems, structural health monitoring, design and testing of wireless RF sensors, and computational electromagnetics. He was a recipient of the 2016 Leopold B. Felsen Award for Excellence in Electromagnetics. Dr. Ozbey was selected as a Fulbright Post-Doctoral Scholar and an URSI Young Scientist Awardee in 2017.

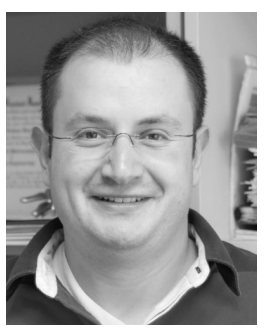

Hilmi Volkan Demir (M'04-SM'11) received the B.Sc. degree in electrical and electronics engineering from Bilkent University, Ankara, Turkey, in 1998 and the M.Sc. and Ph.D. degrees in electrical engineering from Stanford University, Stanford, CA, USA, in 2000 and 2004, respectively. In 2004, he joined Bilkent University, where he is currently a Professor with joint appointments with the Department of Electrical and Electronics Engineering and the Department of Physics. He is also with the Institute of Materials Science and Nanotechnology (UNAM). He is a Fellow of the National Research Foundation in Singapore and a Professor with Nanyang Technological University. His research interests include the development of innovative optoelectronic and RF devices. He was a recipient of the European Union Marie Curie Fellowship, the Turkish National Academy of Sciences Distinguished Young Scientist Award, the European Science Foundation-European Young Investigator Award, and the Nanyang Award for Research Excellence.

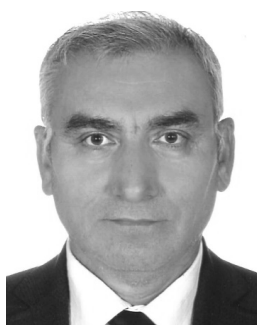

Ayhan Altintas received the B.S. and M.S. degrees from Middle East Technical University, Ankara, Turkey, in 1979 and 1981, respectively, and the Ph.D. degree from The Ohio State University, Columbus, OH, USA, in 1986. From 1981 to 1987, he was with the ElectroScience Laboratory, Ohio State University. He is currently a Professor of Electrical Engineering with Bilkent University, Ankara. He was a Research Fellow and a Guest Professor with Australian National University, Canberra, ACT, Australia, the Tokyo Institute of Technology, Tokyo, Japan, the Technical University of Munich, Munich, Germany, and Concordia University, Montreal, QC, Canada. His current research interests include high-frequency and numerical techniques in electromagnetic scattering and diffraction, propagation modeling and simulation, wireless sensing, and power system monitoring. He is a member of Sigma Xi and Phi Kappa Phi and one of the recipients of the IEEE Third Millennium Medal.

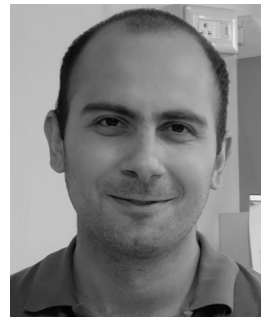

Utku Albostan received the B.S. and M.S. degrees from the Civil Engineering Department, Middle East Technical University (METU), Ankara, Turkey, in 2010 and 2013, respectively, where he is currently pursuing the Ph.D. degree. Since 2012, he has been a Research Assistant with the Civil Engineering Department, METU. His current research interests include digital image correlation technique and its implications on structural systems.

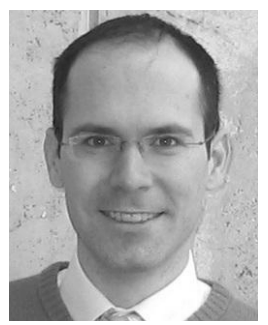

Ozgur Kurc received the B.S. and M.S. degrees in civil engineering from Middle East Technical University (METU), Ankara, Turkey, and the Ph.D. degree in structural engineering from the Georgia Institute of Technology, Atlanta, GA, USA, in 2005. $\mathrm{He}$ is currently a Professor of Structural Engineering with the Structural and Earthquake Engineering Laboratory, Department of Civil Engineering, METU. His current research interests involve wind and earthquake engineering, finite element method, high performance computing, and analysis and design of high-rise buildings.

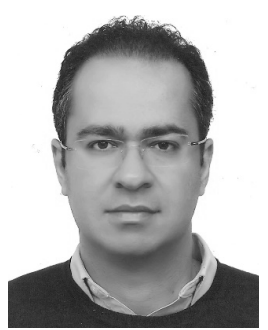

Vakur B. Ertïrk (M'00-SM'17) received the B.S degree in electrical engineering from Middle East Technical University, Ankara, Turkey, in 1993, and the M.S. and Ph.D. degrees from The Ohio State University, Columbus, OH, USA, in 1996 and 2000, respectively. $\mathrm{He}$ is currently a Professor with the Electrical and Electronics Engineering Department, Bilkent University, Ankara. His current research interests include numerical techniques, wireless sensors for structural health monitoring, printed circuits, the analysis and design of conformal antennas and arrays, and scattering from and propagation over large terrain profiles. Dr. Ertürk was the Secretary/Treasurer of the IEEE Turkey Section and the Turkey Chapter of the IEEE Antennas and Propagation, Microwave Theory and Techniques, Electron Devices, and Electromagnetic Compatibility Societies. He was a recipient of the 2005 URSI Young Scientist and the 2007 Turkish Academy of Sciences Distinguished Young Scientist Awards. 